\title{
Measurements of the evaporation rate upon evaporation of thin layer at different heating modes
}

\author{
E.Ya. Gatapova ${ }^{1,2, *}$, E.G. Korbanova ${ }^{1,2}$ \\ ${ }^{1}$ Kutateladze Institute of Thermophysics SB RAS, Novosibirsk, Russia \\ ${ }^{2}$ Novosibirsk State University, Novosibirsk, Russia
}

\begin{abstract}
Technique for measurements of the evaporation rate of a heated liquid layer is presented. The local minimum is observed which is associated with the point of equilibrium of the liquid-gas interface. It is shown when no heat is applied to the heating element temperature in gas phase is larger than in liquid, and evaporation occurs with the rate of $0.014-0.018 \mu \mathrm{l} / \mathrm{s}$. Then evaporation rate is decreasing with increasing the heater temperature until the equilibrium point is reached at the liquid-gas interface. Further increasing of the heater temperature leads to increasing of the evaporation rate.
\end{abstract}

\section{Introduction}

Investigation of evaporation process in a two-layer system under non-isothermal conditions is important for many applications, such as microelectronics cooling [1]. The advancement in high performance heat exchangers, vapor chambers and heat pipes [2] with microscale passages stimulates also development of a new fundamental knowledge of the processes with phase change $[3,4]$.

If the liquid and the vapor-gas mixture are stationary it is usually considered that the movement of the vapor in the gas phase occurs mainly as a result of molecular diffusion. However, if the molecular mass of the evaporating liquid is less than that of the gas contacting with the liquid, then the moment comes when the buoyancy forces remove the gas layer from equilibrium state and the diffusion regime is replaced by a convective one. The resulting convection can significantly increase the evaporation rate [5]. Investigations of the temperature profile near liquid-gas interface and the conditions when the equilibrium state at the interface is reached for complex system are important from the practical and fundamental point of views. Evaporation occurs under non-equilibrium conditions and the continuity of the temperature at the interface can not be ensured. Evaporative flux depends on the thermal difference at the interface [4], and the precise measurements of these two quantities (temperature and evaporation rate) can give information on the boundary

\footnotetext{
* Corresponding author: gatapova@itp.nsc.ru
} 
condition at the liquid-gas interface as well as data on the evaporation/condensation coefficients.

In this work, we present the results of the evaporation rate measurements of the locally heated liquid layer.

\section{Experimental setup and measurements technique}

Experiments are conducted at normal atmospheric condition. Ultrapure deionized and degased water (MilliQ) is used as a working fluid for experiments. The liquid is injected using a syringe pump (Cole Parmer) to cuvette with local heater, which is placed in microbalance system (Fig. 1). Calculation of the mass of evaporated liquid is carried out using BM-252 microbalance of accuracy class I (special), with the smallest weighing limit of $0.01 \mathrm{mg}$ and control of the ambient gas humidity and temperature. Microbalance is connected to PC and measurements are controlled by special software. The experiments are performed at quasi-stationary heating mode. The temperature of the heater is controlled by three thermocouples. The pressure is measured by barometer. Calibrations of all thermocouples are carried out in the temperature range $5-100{ }^{\circ} \mathrm{C}$. Two reference thermometers (ETS-100) are used. Data registration is carried out by means of temperature control and measurement system consisting of data collection (NI 9214) and the corresponding software.

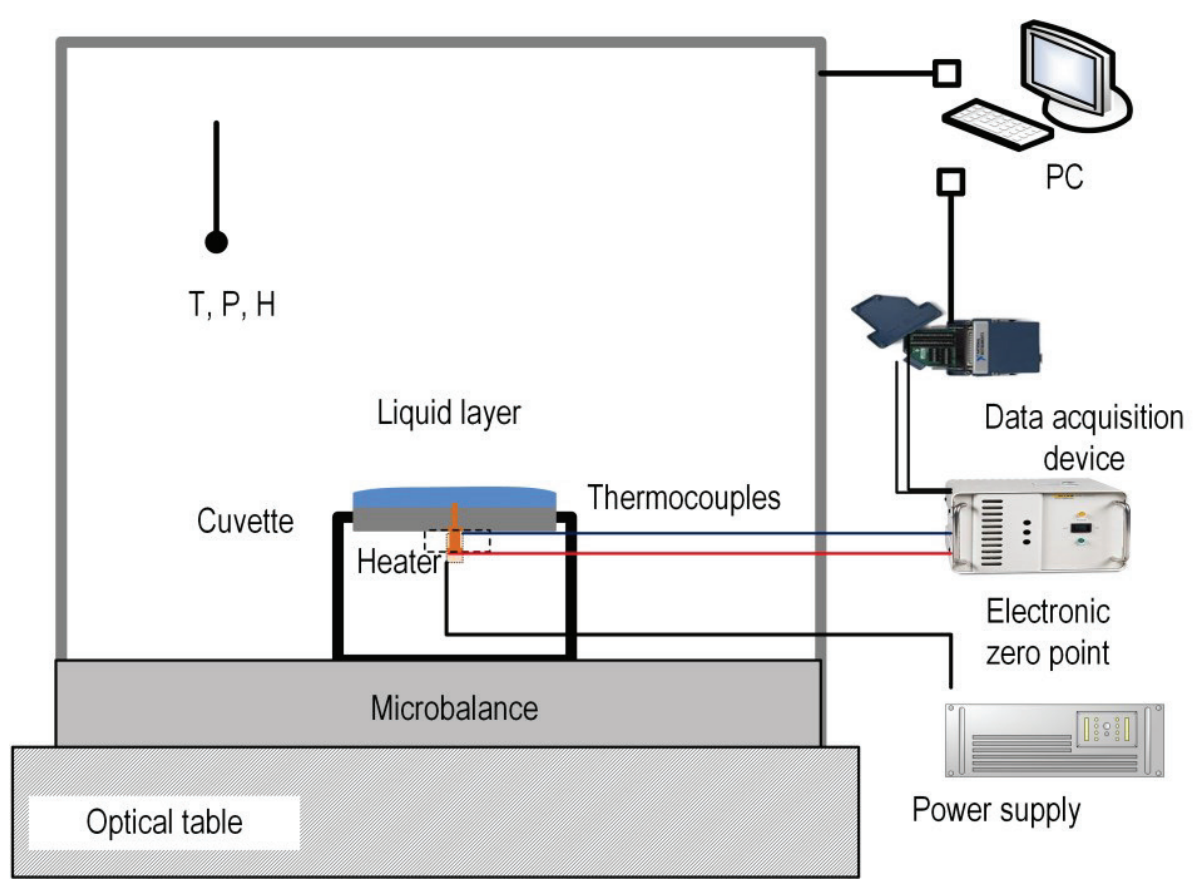

Fig. 1. Scheme of the experimental setup. 


\section{Results and discussion}

Results of the evaporation rate measurements for water-air system are presented in Fig. 2. The local minimum is observed which is connected with the point of equilibrium of the liquid-gas interface. Let us discuss the data on the temperature profile across water-air system near interface obtained in Ref. [4]. When no heat is applied to the heating element the negative value of the temperature jump is measured, i.e. the temperature in the air near the liquid-gas interface is larger than in liquid. By increasing of the heat flux in heater some kind of equilibrium point can be observed, where temperature jump is absent. The measured temperature profiles have the classical continual shape. The fracture in the temperature profile is observed, which is due to different thermal conductivities of liquid and gas phases. By further increasing of heat flux in heater, the slope of the temperature profile becomes steeper, and thus the temperature gradient and Fourier heat fluxes in gas and liquid phases. The further increase of the heating leads to increase of the temperature jump and evaporation rate [4].

Basing on our previous temperature profile measurements [4], we can conclude that when no heat is applied to the heating element temperature in gas phase is larger than in liquid, and evaporation occurs with the rate of $0.014-0.018 \mu \mathrm{l} / \mathrm{s}$. Then evaporation rate is decreasing with increasing heater temperature until the equilibrium without temperature jump is reached at the liquid-gas interface. Further increasing of the heater temperature leads to increasing of the evaporation rate.

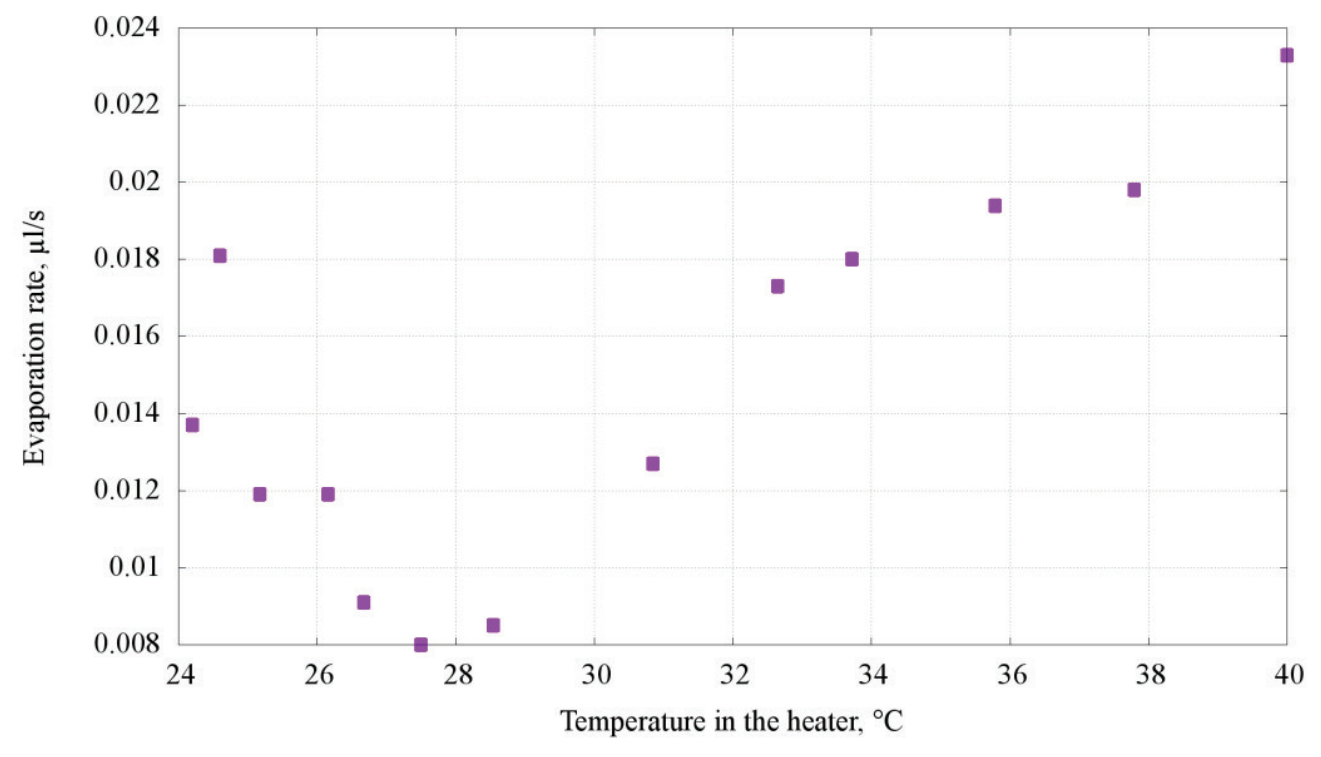

Fig. 2. Evaporation rate dependence on the average temperature of the heater.

In conclusion, results of the evaporation rate measurements of a heated liquid layer are presented. It is shown that there is local minimum of the evaporation rate at the point of equilibrium of the liquid-gas interface. 
The authors gratefully acknowledge the support of this work by the Ministry of Education and Science of the Russian Federation (Agreement 14.616.21.0016, project identifier RFMEFI61614X0016).

\section{References}

1. M.H. Nasr, C.E. Green, P.A. Kottke, X. Zhang, T.E. Sarvey, Y.K. Joshi, M.S. Bakir, A.G. Fedorov, International Journal of Heat and Mass Transfer, 108, 1702 (2017)

2. M.A. Chernysheva, S.I. Yushakova, Y.F. Maydanik, International Journal of Heat and Mass Transfer, 81, 297 (2015)

3. E.Y. Gatapova, I.A. Graur, F. Sharipov, O.A. Kabov, International Journal of Heat and Mass Transfer, 83, 235 (2015)

4. E.Y. Gatapova, I.A. Graur, O.A. Kabov, V.M. Aniskin, M.A. Filipenko, F. Sharipov, L. Tadrist, International Journal of Heat and Mass Transfer, 104, 800 (2017)

5. L. Prandtl, O. Tietjens, Applied hydro- and aeromechanics, Dover publications, inc. (1934) 